Dossier Interdisciplinarité

\title{
Territoire : agronomie, géographie, écologie, où en est-on ? Le point de vue d'un chercheur agronome
}

\author{
Jean Boiffin \\ Agronome, INRA, Direction scientifique Agriculture, activités, territoires, 147 rue de l’Université, 75338 Paris cedex 07, France
}

Le territoire a-t-il été, et est-il aujourd'hui, un sujet de rencontre et de collaboration entre agronomes, écologues et géographes ${ }^{1}$ ? Précisons tout d'abord le domaine scientifique et technique auquel se réfèrent les quelques éléments de réponse qui suivent. Sous le vocable agronomie, nous entendons ici la discipline qui étudie les relations entre le peuplement végétal, le milieu et les interventions qui leur sont appliquées, en vue de définir des modalités de gestion durable des espaces cultivés. De façon expéditive et si l'on s'en tient, d'une part, à cette définition « restreinte » de l'agronomie, d'autre part à une acception du terme territoire elle aussi plus exigeante et restrictive qu' «espace géré», la réponse est plutôt négative. Au-delà des travaux pionniers de J.-P. Deffontaines, au-delà de références au territoire de plus en plus souvent exprimées dans les travaux des agronomes, on ne peut faire état d'un flux significatif et régulier de publications scientifiques, de projets de recherche et, a fortiori, de structures de collaboration qui répondent à des critères objectifs d'implication conjointe de ces communautés autour de la notion de territoire. L'analyse de cette situation conduit à prévoir qu'elle devrait se modifier dans le sens d'une interaction accrue, puis à proposer quelques pistes de travail qui pourraient être un support de rapprochement entre les trois communautés scientifiques.

\section{Les raisons d'une faible interaction}

Sans chercher à les classer ou à les ordonner, ni surtout à être exhaustif, on peut avancer quelques éléments

\footnotetext{
Auteur correspondant : Jean.Boiffin@paris.inra.fr

1 Ce texte reprend les éléments d'une intervention orale faite dans le cadre des Entretiens du Pradel «Agronomes et Territoires » en septembre 2002.
}

explicatifs, les uns plutôt de conjoncture scientifique, les autres relevant du contexte socioéconomique et politique qui a conditionné l'évolution de l'agronomie dans les deux ou trois dernières décennies.

Dans la première catégorie, on relèvera tout d'abord le souci, pour l'agronomie, d'échapper à la qualification de «science de localité ». Il a incité la recherche à se concentrer sur l'étude de processus invariants quel que soit le lieu, et à dé-régionaliser son dispositif. A contrario, les approches à caractère régional suscitaient, de la part des chercheurs, la crainte de s'enliser dans une infinité d'études de cas à caractère monographique. Ces réticences en partie justifiées ont probablement contribué à masquer l'intérêt, si ce n'est l'existence même, de sujets de recherche sur les territoires et les dynamiques territoriales, qui justement avaient une portée générique. En sens inverse, on notera aussi la faible sollicitation externe de l'agronomie sur ces sujets de recherche: ceux qui, parmi les géographes et/ou les écologues du paysage, ont développé ou utilisé la notion de territoire n'ont guère fait appel aux agronomes. On peut aisément s'en convaincre en consultant la bibliographie des ouvrages de référence en la matière. On peut d'ailleurs se demander si le souci légitime d'échapper au ruralisme n'a pas conduit leurs auteurs à l'excès inverse : une certaine sous-estimation de la place des systèmes de production agricole et de leurs évolutions dans les dynamiques territoriales et, en leur sein, dans les dynamiques écologiques.

Mais un facteur de cloisonnement majeur a sans doute été le poids du productivisme agricole sur l'agronomie et les recherches en agronomie - tout au moins en métropole. On veut dire par là, non pas que les chercheurs en agronomie ont été des militants du productivisme, mais que leurs orientations de recherche ont été marquées par 
la primauté de la finalité de production, avec un certain nombre de corollaires « anti-territoriaux ». Citons-en trois :

- le fait que les interactions entre agriculture et autres activités aient été plutôt appréhendées dans le cadre de filières que dans le cadre de territoires. Cela se traduit notamment, dans la structure des partenariats de recherche-développement et d'application, par la prédominance des collaborations avec les instituts techniques de filières;

- la prépondérance, dans les approches de terrain, des niveaux d'organisation "station » et " parcelle », c'est-àdire des entités spatiales les plus homogènes vis-à-vis des différents facteurs de production ;

- l'occultation par l'usage intensif des intrants, en particulier phytosanitaires, de processus à caractère fortement spatial, dont la prise en compte aurait immanquablement amené les agronomes à travailler avec des écologues et des géographes, notamment à l'échelle du paysage : ainsi, l'épidémiologie des bio-agresseurs des cultures était en quelque sorte rendue sans objet, dans un contexte de traitement chimique préventif et spatialement uniforme.

\section{Les évolutions envisageables}

Cette situation de relativement faible interaction autour de la notion de territoire va-t-elle se modifier? Là encore, sans prétendre à l'exhaustivité, on peut indiquer trois moteurs d'évolution des problématiques de l'agronomie, dont le point commun est de rendre nécessaires des approches pluri- ou transdisciplinaires des activités agricoles, et de leur insertion dans des territoires ou des dynamiques territoriales.

Un premier est la nécessité de traiter les problèmes d'environnement liés à l'agriculture, qu'elle en soit cause, victime ou gestionnaire potentiel. Dans de nombreux cas - de façon exemplaire celui de la qualité de l'eau -, il s'avère que leur résolution ne relève pas seulement des bonnes pratiques agricoles. On entend par là un ensemble de mesures subordonnées aux finalités de production (incluant des mesures de protection directe de la santé des agriculteurs et des consommateurs), et mises en œuvre au niveau des parcelles, des troupeaux ou des bâtiments. Au-delà de ces mesures, qui se cantonnent à une logique de respect ou de préservation de l'environnement, il faut recourir à une gestion agri-environnementale qui soit à la fois dédiée à l'environnement, intégrée et spatialisée. Cette gestion implique en effet des aménagements ou mesures spécifiques sur des sites appropriés. De surcroît, elle s'applique à des entités spatiales qui ne coïncident pas avec les parcelles ou exploitations agricoles, et sont souvent beaucoup plus larges, l'exemple évident étant celui du bassin hydrologique. Elle doit donc être collective, et être conçue et mise en œuvre de concert avec d'autres occupants ou acteurs que l'agriculture elle-même : on a bien là certains des ingrédients d'une gestion non seulement spatiale, mais territoriale au sens le plus exigeant du terme.

Même s'ils se cantonnaient au cadre de l'agriculture raisonnée et des bonnes pratiques agricoles, les agronomes seraient de toute façon confrontés à un deuxième moteur de «territorialisation » et «d'écologisation » de l'agronomie : les restrictions croissantes, pour des raisons à la fois économiques et réglementaires, à l'usage des intrants d'origine industrielle, et tout particulièrement des pesticides. Ces restrictions appellent l'élaboration de méthodes dites de protection intégrée basées sur la connaissance et la maîtrise des processus spatiotemporels de développement des populations de parasites, de ravageurs et d'adventices. Elles appellent aussi à constituer les bases d'une gestion spatiale raisonnée, à l'échelle régionale si ce n'est continentale ou planétaire, des innovations variétales ou phytosanitaires, de façon à éviter ou retarder soit le contournement des résistances variétales, soit l'apparition précoce de résistances aux produits phytosanitaires. On retrouve cette nécessité d'approches conjointes d'agronomie, d'écologie du paysage et de biogéographie pour analyser l'impact de la dissémination des organismes génétiquement modifiés : ce point est illustré par les collaborations exemplaires qui se sont nouées récemment entre agronomes et écologues sur la modélisation des flux de gènes dans les agro-écosystèmes.

Plus globalement, un troisième moteur de rapprochement entre agronomes, écologues et géographes, autour ou dans le cadre de la notion de territoire, est la remise en question des fondements de la politique agricole commune (PAC) et des critères d'attribution des soutiens publics à l'agriculture. Cette remise en question n'est d'ailleurs que la partie la plus apparente d'une redéfinition du statut de l'agriculture dans les sociétés développées, dont les corollaires sont le découplage des aides directes par rapport aux quantités produites, l'instauration d'une certaine dose d'éco-conditionnalité, la baisse des prix unitaires des produits de masse, et la réorientation d'une partie des soutiens vers le développement rural. La question qui se pose alors est celle d'un éventuel recouplage des soutiens publics à des fonctions autres que la production, notamment environnementales et territoriales, qui pourraient être remplies par l'agriculture conjointement avec celle de production. Il faut alors s'assurer de la possibilité réelle d'exercice par l'agriculteur de ces fonctions autres (c'est le débat entre multifonctionnalité de l'agriculture et multifonctionnalité de l'espace rural), et donc de leur possibilité d'intégration aux systèmes de culture et de production : quelles pratiques, quels systèmes, quelle agronomie pour les fonctions non productives de l'agriculture, par exemple des fonctions 
de recyclage des produits résiduaires d'origine urbaine ou industrielle, de séquestration du carbone, de gestion de la biodiversité, de prévention des catastrophes naturelles, etc. ?

\section{Quelques pistes pour un travail conjoint}

Aucune de ces évolutions vitales pour l'agronomie ne peut être assumée par elle toute seule, et ceci presque par définition. Il faut dès lors souhaiter qu'agronomes, écologues et géographes prennent conscience de leurs intérêts mutuels à un travail en commun, et pour cela qu'ils identifient des questions de recherche communes. On peut suggérer que chaque communauté scientifique y contribue en proposant des «questions candidates ", à la fois importantes pour elle-même et susceptibles d'intéresser les autres. Â titre d'ébauche pour le compte des agronomes, on avance ci-dessous trois exemples:

1. Comment évolue et va évoluer la localisation des systèmes de production agricole, en fonction des déterminants majeurs que sont les politiques agricoles et les marchés, mais aussi les politiques environnementales, les autres changements d'occupation des sols, les changements climatiques et écologiques ? Quels sont les impacts écologiques, économiques et sociaux des déplacements en cours et à venir?

2. Dans quels systèmes d'interaction avec les autres activités et occupations de l'espace les exploitations agricoles se trouvent-elles, en fonction de leur localisation et de leurs caractéristiques technico-économiques? Ces interactions jouent-elles ou peuvent-elles jouer un rôle actif vis-à-vis de certaines dynamiques territoriales positives ou négatives? On pense, dans notre contexte européen, aux interactions agriculture-tourisme, à l'agriculture péri-urbaine, aux interactions forêt-agriculture. On pense aussi aux interactions agriculture-agriculture, par exemple celles entre systèmes de production végétale et systèmes d'élevage sur lesquelles G. Lemaire nous invite à porter un nouveau regard, dans le cadre élargi d'une approche territoriale ${ }^{2}$. On devra aussi, en prolongement des questions précédentes, se demander comment ces diverses interactions sont amenées à se modifier, compte tenu de l'évolution de l'occupation du territoire, et dans un contexte de changement global, en particulier climatique.

3. Quelles sont les conséquences de ces interactions pour l'agriculture et ses pratiques, en termes de contraintes ou d'opportunités? Dans quelle mesure les couverts végétaux, leur conduite et leur répartition spatiale peuvent-ils être utilisés, non seulement en tant que variables d'ajustement comme le sont actuellement les jachères, mais aussi comme outils d'aménagements et de gestion de l'espace? Peut-on concevoir une innovation agronomique à visée écologique et territoriale, spécifiquement adaptée, par exemple, à des contextes périurbains ou de transition prairie-forêt, à des finalités de dépollution, de gestion spatialisée des cycles biogéochimiques, de construction et d'entretien de paysages? En cas de réponse positive, quelles peuvent être les modalités d'expression et de valorisation de ces systèmes et pratiques agri-environnementaux au plein sens du mot, en termes de cahiers des charges, de contrats individuels ou collectifs, de clauses dans les baux ruraux ou transactions foncières, etc. Les difficultés et, surtout, les malentendus qui compromettent la mise en œuvre des CTE conduisent à insister sur le fait que l'objectif n'est pas ici un dispositif de relégitimation des aides, mais bien la définition et la mise en œuvre concrète de fonctions et services territoriaux - y compris, dans certains cas, de gestion écologique - justifiant objectivement une rémunération appropriée.

Cette esquisse de questionnement est beaucoup trop incomplète, insuffisamment hiérarchisée et peu affinée pour attendre des communautés interlocutrices de l'agronomie un écho explicite et immédiat. Plus encore que des sujets identifiés, on a souhaité par là leur suggérer une démarche de rapprochement qui partirait de questions partagées, plutôt que de lieux ou de régions qui seraient arbitrairement fixés comme terrains d'études communs, même si, in fine, il faut bien se placer quelque part pour collaborer sur de telles questions.

\footnotetext{
${ }^{2}$ Allusion à l'exposé de G. Lemaire au cours du même
} séminaire. 